\title{
Commentary: Description of the Position and Performance of an Echocardiogram by Subcostal View During the Prone Position in A Patient with Severe Pneumonia Caused by COVID-19
}

\author{
César Del Castillo Gordillo',2*, Mario Alfaro Diaz \\ 'Cardiovascular center, San Borja Arriaran Hospital, Santiago, Chile \\ ${ }^{2}$ Cardiovascular center, DIPRECA Hospital, Santiago, Chile
}

\section{Article Info}

\section{Article Notes}

Received: December 31, 2020

Accepted: January 19, 2021

\section{${ }^{*}$ Correspondence:}

Dr. César Del Castillo Gordillo, MD, Cardiologist,

Cardiovascular center, DIPRECA Hospital, 1200

Vital Apoquindo, 7601003, Santiago, Chile; Email:

cesardelcastillogor@gmail.com.

(c) 2021 Del Castillo Gordillo C. This article is distributed under the terms of the Creative Commons Attribution 4.0 International License.

\section{Keywords:}

COVID-19

Echocardiography

Mechanical ventilation

Prone position
The methods to hemodynamic monitoring in critically ill patients subjected to the prone position during invasive mechanical ventilation have been discussed for some time. In imaging studies in patients who are meant to undergo radiotherapy, the heart moves anteriorly about $2 \mathrm{~cm}$ towards the chest wall, and there is a decrease in the interposed lung volume from $15 \mathrm{~mL}$ in the supine to $3 \mathrm{~mL}$ in the prone position ${ }^{1}$, a factor that could favor the performance of a transthoracic echocardiogram in the prone position. Studies in the operating room have begun to show that there is a possibility of performing echocardiographic assessment in patients who require a prone position during surgery, mainly for the assessment of the inferior vena cava (IVC) ${ }^{2}$. Subsequently, the assessment in critically ill patients started with the description of the swimmer's position, which consisted of flexing the patient's left arm, followed by placing a pillow under the same arm, and then acquiring the apical images at the level of the maximum cardiac impulse zone ${ }^{3}$. In patients not on invasive mechanical ventilation, the feasibility of evaluating the left ventricle (LV) through its ejection fraction and longitudinal global strain was archived ${ }^{4}$. Another analysis, also in patients not undergoing invasive mechanical ventilation, but aimed at analyzing the right ventricle (RV) in 50 patients, showed that systolic function parameters such as tricuspid annulus plane systolic excursion (TAPSE) could be obtained in $100 \%$ of their cases 5 . On the other hand, the evaluation of the IVC and the state of volemia has been evaluated in patients who required invasive mechanical ventilation and prone position during surgery, but from the right side of the patient ${ }^{2}$. These studies demonstrated that the echocardiogram in the prone position was possible and of clinical utility.

During the COVID-19 pandemic, published research in this field has increased, however these studies are a small sample size. Gibson et $\mathrm{al}^{6}$, described 27 patients in the swimmer's position with invasive mechanical ventilation and COVID-19 pneumonia, achieving apical 4-chamber vision in all, apical 5-chamber view in 96\% (26/27), and IVC in $92.5 \%$ (25/27). Quantitative evaluation of LV function was obtained in $96 \%(26 / 27)$ of cases through mitral annulus plane systolic excursion (MAPSE), velocity-time integral (VTI) of the LV outflow tract (LVOT), and estimation of filling pressures. Likewise, assessment of RV function was achieved in 88.8\% (24/27) of cases through TAPSE, S' wave of the tricuspid annulus, and RV size. García et $\mathrm{al}^{7}$, described 15 patients positioned with the left arm flexed, but without a pillow under it. Analysis of the LV function was done through 
its qualitative evaluation, VTI LVOT, MAPSE, and anterolateral and infero-septal segments by the visualization of apical 4 and 5 chambers, while analysis of the RV function was performed through its basal diameters, TAPSE, and S 'wave of the tricuspid annulus. Functional evaluation of the $\mathrm{LV}$ was achieved in all cases and of the RV in 93\% (14/15) of those analyzed (in one patient, no free wall was seen). Furthermore, Gibson et al's ${ }^{6}$ protocol implied displacing the operator and echocardiography machine to perform the evaluation of the IVC by the right lateral view, which was achieved in $92.5 \%$ (25/27) of the study subjects.

The influence of mechanical ventilation variables on image quality when performing an echocardiogram is widely known. However, Gibson et $\mathrm{al}^{6}$ indicated that $92.5 \%$ of their patients had moderate levels of positive pressure at levels where the end of expiration was $\geq 10 \mathrm{~cm} \mathrm{H}_{2} \mathrm{O}$.

Currently, variations of the supine and prone positions in terms of cardiovascular function have not been fully clarified. A retrospective study by Giustininano et $\mathrm{al}^{8}$ analyzed 8 patients on invasive mechanical ventilation due to COVID-19 pneumonia. Their findings were only described in the apical 4-chamber vision, where it showed that $75 \%(6 / 8)$ of the patients did not changes the $\mathrm{LV}$ ejection fraction, and $62.8 \%(5 / 8)$ did not change the basal diameter of the RV and pulmonary artery systolic pressure. Santos-Martinez et $\mathrm{al}^{5}$ analyzed 50 patients without invasive mechanical ventilation and found that RV function tends to improve with statistically significant results through the measurement of TAPSE, $S$ 'wave of the tricuspid annulus, and fractional area change. In another study, Hemsley et $\mathrm{al}^{2}$ failed to describe a significant change in the size of the IVC in the supine versus prone position, with a tendency to maintain a similar size or increase.

A complement to what has been described is the use of pulmonary ultrasound, which is a tool for monitoring and evaluating the severity of pneumonia and/or acute respiratory distress in patients on mechanical ventilation and in prone position?.

However, despite the description of the apical view and the great amount of information obtained through them, there is a limitation in the quality of images in critical patients, especially those on mechanical ventilation. This does the search for alternative ways to assess cardiovascular function in these patients via transthoracic echocardiography of utmost importance.

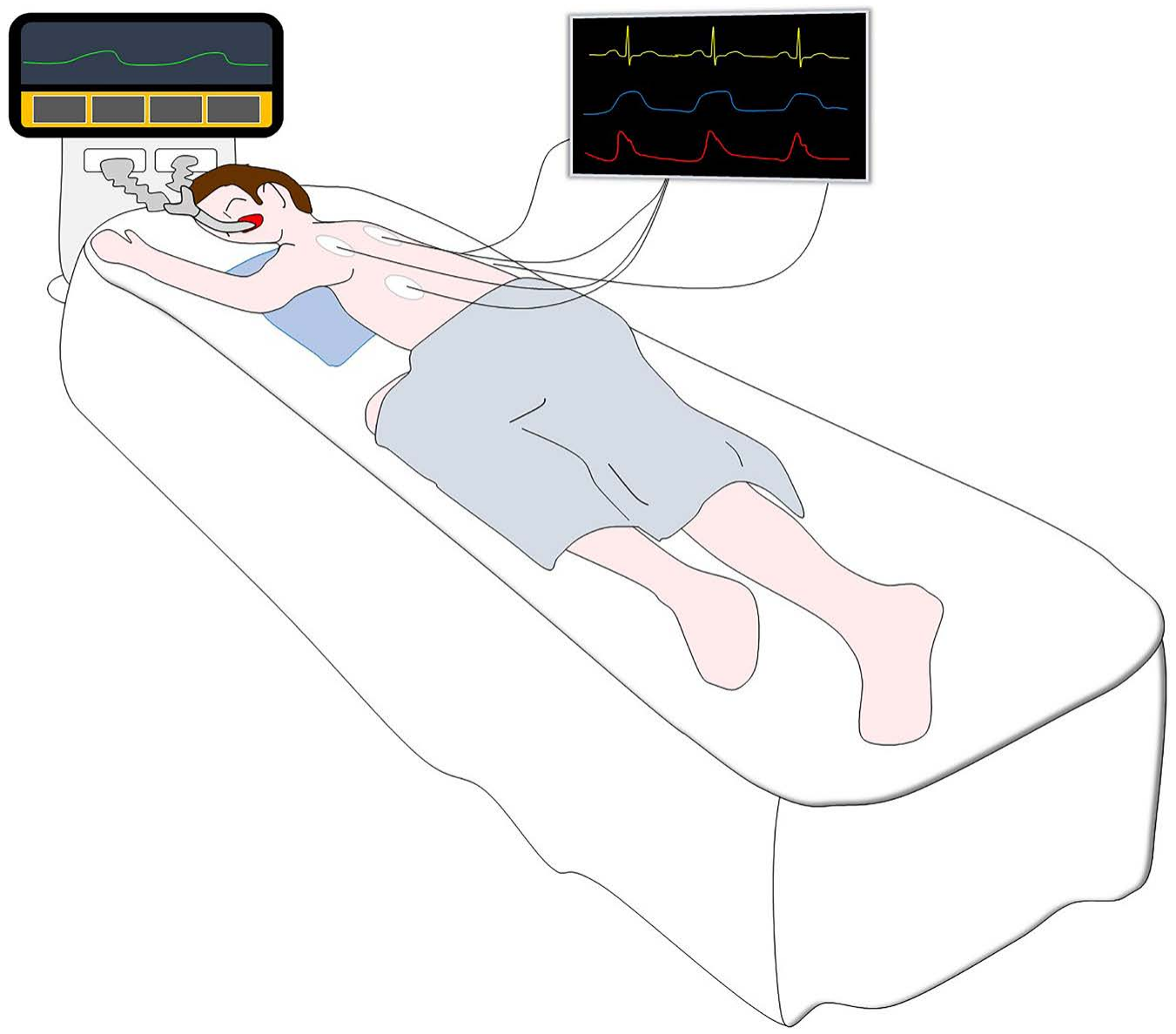

Figure 1. Position to perform echocardiogram in prone position during invasive mechanical ventilation. 
Given this, our group has published a complementary view $^{10}$. In patients in the prone position during invasive mechanical ventilation, through a modification of the position described above, we placed a pillow under the patient's left hemithorax and raised the left upper limb with a flexed elbow; the apical window was better exposed and allowed space for a subcostal window (Figure 1). In this way, we were able to successfully perform a 4-chamber subcostal vision assessment, RV outflow tract vision, and IVC assessment. We were able to assess the bi-ventricular and valve function through subcostal 4-chamber vision, add to the volume status with the assessment of the IVC. In addition, important information on a possible pericardial disease and presence of pulmonary hypertension was added through measurement of the acceleration time at the level of the RV outflow tract and the shape of its curve (using pulsed doppler). Also, it was possible to evaluate the presence of a tricuspid regurgitation gradient and the function of the pulmonary valve, which may be important in different cardiological diseases such as congenital heart disease.

Among the advantages of this position is that we achieve apical and subcostal vision from the same side and position of the patient, reducing reasoning and exposure times for the health professional. Moreover, this position is easy to acquire by the health team that cares for the patient in the prone position and without additional risk to the patient.

We have complemented this with the use of apical window in $5,4,2$, and 3 chambers $^{11}$; therefore, we think that the modification to the position, as we have described, and an apical-subcostal echocardiogram protocol could offer an improvement in the evaluation of cardiovascular function in critically ill patients in the prone position during invasive mechanical ventilation.

\section{References}

1. Chino JP, Marks LB. Prone positioning causes the heart to be displaced anteriorly within the thorax: implications for breast cancer treatment. Int J Radiat Oncol Biol Phys. 2008; 70: 916-20.

2. Hensley J, Wang H. Assessment of volume status during prone spine surgery via a novel point-of-care ultrasound technique. Cureus. 2019; 11: e4601.

3. Ugalde D, Medel JN, Romero C, et al. Transthoracic cardiac ultrasound in prone position: a technique variation description. Intensive Care Med. 2018; 44: 986-7.

4. Roemer S, Kaminski A, Payne A, et al. Feasibility of transthoracic imaging of the heart in the prone position. J Am Soc Echocardiogr. 2020 ; 33(9): 1147-48.

5. Santos-Martínez L, Mendoza-Copa G, García-Cruz E, et al. Factibilidad en la estimación ecocardiográfica de parámetros del ventrículo derecho en posición prono. Arch Cardiol Mex. 2020; 90(2): 116-23.

6. Gibson LE, Di Fenza R, Berra L, et al. Transthoracic echocardiography in prone patients with acute respiratory distress syndrome: a feasibility study. Crit Care Explor. 2020; 2: e0179.

7. Garcia-Cruz E, Manzur-Sandoval D, Gopar-Nieto R, et al. Transthoracic echocardiography during prone position ventilation: lessons from the COVID-19 pandemic. JACEP Open. 2020; 1-7.

8. Giustiniano E, Fazzari F, Bragato RM, et al. Trans-thoracic echocardiography in prone positioning COVID-19 patients: a small case series. SN Compr Clin Med. 2020; 1-6.

9. Koenig S, Mayo P, Volpicelli G, et al. Lung ultrasound scanning for respiratory failure in acutely ill patients. CHEST 2020; 158(6): 251116.

10. Del Castillo Gordillo C, Yañez Vidal F, Begazo Gonzales A, et al. Description of the position and performance of an echocardiogram by subcostal view during the prone position in a patient with severe pneumonia caused by COVID-19. BMJ case rep. 2020; 13: e239230.

11. Del Castillo C, Yañez F, Begazo A, et al. Protocolo de ecocardiograma transtorácico apical-subcostal en posición prono durante ventilación mecánica invasiva. LVI Congreso Chileno de cardiología y cirugía cardiovascular online. 2020; Dec 02-05; Chile; Available from: http:// www.sochicar.cl/images/Eventos/2020/noviembre/REVISTACONGRESO-2020.pdf. 